\title{
Diagnóstico de gestação em cabras da raça Saanen através do uso do efeito Doppler e da ultra-sonografia em tempo real
}

\section{Pregnancy diagnosis in Saanen goats by Doppler effect and the real time ultrasonography}

Ney Rômulo de Oliveira Paula, ${ }^{\star}$ Jurandir Ferreira da Cruz, ${ }^{\star \star}$ Edilson Soares Lopes Júnior, ${ }^{\star}$ Dárcio Ítalo Alves Teixeira,
Juliana Bezerra Lima-Verde, ${ }^{\star}$ Davide Rondina, ${ }^{\star}$ Vicente José de Figueirêdo Freitas ${ }^{\star}$

\section{Resumo}

Com o objetivo de verificar a eficiência do efeito Doppler como método de diagnóstico precoce da gestação em caprinos, 13 cabras da raça Saanen foram monitoradas aos 30,45, 60, 75, 90, 105 e 120 dias de gestação, utilizando-se um equipamento baseado no efeito Doppler e comparando-se à ultra-sonografia em tempo real. Quanto ao percentual de acerto para gestação positiva, foram verificadas diferenças estatísticas $(P<0,05)$ entre os métodos aos $30,45,60,75$ e 105 dias de gestação, sendo que a ultra-sonografia em tempo real sempre apresentou resultados superiores. Comparando-se a eficiência do efeito Doppler, entre as idades gestacionais, verificou-se um baixo percentual de acerto aos $30(0 \%)$ e $45(22,2 \%)$ dias de gestação, aumentando este percentual a partir dos 60 dias pós-monta (66,7\%). Para esse parâmetro, quando do uso do efeito Doppler, foram verificadas diferenças estatísticas $(P<0,05)$ aos $90(55,6 \%$ vs $22,2 \%)$ e aos $120(55,6 \%$ vs $33,3 \%)$ dias de idade gestacional em cabras com gestação múltipla ou simples, respectivamente. Observou-se uma maior freqüência cardíaca materna nas cabras com gestação múltipla aos 105 e 120 dias de idade gestacional, quando comparadas com as de gestação simples. Finalmente, quando da comparação com a ultra-sonografia em tempo real, o uso do efeito Doppler mostrou-se ser um método eficaz para o diagnóstico de gestação em caprinos a partir dos 60 dias pós-fecundação.

Palavras-chave: caprino, efeito Doppler, ultra-sonografia, diagnóstico de gestação.

\section{Abstract}

The aim of this work was to verify the efficiency of Doppler effect as a method for early pregnancy diagnosis in goats. Thus, 13 Saanen goats were monitored at 30,45,60, 75, 90, 105 and 120 days of pregnancy, using the Doppler effect and real time ultrasonography. Concerning the percentage of accuracy for positive pregnancy between methods at 30, 45, 60, 75 and 105 days of pregnancy, the ultrasonography was always more efficient than Doppler effect $(P<0.05)$. Comparing the Doppler effect efficiency, between different days of pregnancy, it was verified a low percentage at $30(0 \%)$ and $45(22.2 \%)$ days after mating, but this percentage rised from 60 days of pregnancy $(66.7 \%)$. For this parameter and using the Doppler effect, significant differences $(P<0.05)$ were verified at $90(55.6 \%$ vs $22.2 \%)$ and at $120(55.6 \%$ vs $33.3 \%)$ days of pregnancy in goats with multiple or single pregnancy, respectively. It was observed an higher maternal cardiac frequency in goats with multiple pregnancy at 105 and 120 days after mating, when compared to single pregnancy. Finally, when comparing to real time ultrasonografy, the use of Doppler effect showed to be an efficient method for pregnancy diagnosis in goats from 60 days after fertilization.

Keywords: goat, Doppler effect, ultrasonography, pregnancy diagnosis.

\section{Introdução}

O desenvolvimento de biotécnicas da reprodução tem permitido o aumento da produtividade dos rebanhos. Neste sentido, o diagnóstico precoce de gestação tem grande importância econômica e prática, pois além de minimizar os custos adicionais com a suplementação alimentar de fêmeas vazias, facilita o manejo reprodutivo e auxilia o processo de seleção, na medida em que identifica as fêmeas com problemas de fertilidade. $O$ diagnóstico precoce de gestação em caprinos apresenta as mesmas vantagens observadas em outras espécies domésticas. No entanto, para os pequenos ruminantes, esta técnica apresenta dificuldades em função da limitação anatômica para a avaliação do sistema genital através da palpação retal.

Em caprinos, diversos métodos de diagnóstico precoce de gestação têm sido utilizados, como por exemplo: controle de retorno ao estro (Engeland et al., 1997), palpação reto-abdominal (Ott et al., 1981), biópsia vaginal (Ishwar, 1995), dosa-

* Universidade Estadual do Ceará, Av. Paranjana, 1700, Fortaleza - CE - vjff@uece.br.

** Universidade Estadual do Sudoeste da Bahia. 
gem de sulfato de estrona (Refstal et al., 1991), dosagem de progesterona (Engeland et al., 1997), determinação da proteína associada à gestação (PAG) (Sousa et al., 1999), laparoscopia (Greyling et al., 2002), palpação do útero por laparotomia (Smith, 1980), radiografia (Barker e Cawley, 1967) e ultra-sonografia em tempo real (Bretzlaff et al., 1993). No entanto, muitos desses métodos de diagnóstico apresentam limitações para utilização em propriedades rurais, em função do elevado custo do equipamento, da necessidade de mãode-obra especializada, bem como da baixa precisão daqueles de fácil aplicação.

A técnica de ultra-sonografia em tempo real, quando comparada com as outras técnicas disponíveis, apresenta diversas vantagens no diagnóstico de gestação. É uma técnica rápida, segura, prática e de alta precisão para determinar a idade gestacional (Doize et al., 1997), o número de fetos (Russel, 1990) e avaliar a viabilidade fetal (Ishwar, 1995). Contudo, a ultra-sonografia em tempo real apresenta um custo bastante elevado e necessita de mão-de-obra qualificada, o que a torna bastante limitada para as situações de rotina a campo.

O efeito Doppler tem sido utilizado mundialmente para o diagnóstico precoce de gestação na espécie humana (Callahan et al., 1964), bem como em caprinos e ovinos (Ishwar, 1995). No entanto, apesar deste aparelho apresentar baixo custo, a sua utilização ainda é limitada em rebanhos caprinos, justificando-se desta forma o desenvolvimento de estudos que possam avaliar a precisão deste equipamento. Este trabalho teve como objetivo avaliar a eficiência do efeito Doppler (DPPR-80) para o diagnóstico precoce de gestação em caprinos, quando comparado à ultra-sonografia em tempo real.

\section{Material e métodos}

O experimento foi realizado no setor de Caprino e Ovinocultura da Universidade Estadual do Ceará, localizada na cidade de Fortaleza. Foram utilizadas 14 cabras adultas da raça Saanen com peso e idade média ( \pm d.p.) de $48,6 \pm 7,9 \mathrm{~kg} \mathrm{e} 6,7 \pm 1,7$ anos, respectivamente. Durante o experimento, todas as cabras foram mantidas em sistema intensivo, sendo alimentadas com capim elefante (Pennisetum purpureum) e suplementadas com concentrado contendo $20 \%$ de proteína bruta. Água e sal mineral foram fornecidos ad libitum.

As cabras foram submetidas a um tratamento de sincronização do estro através do uso de esponjas vaginais impregnadas com $45 \mathrm{mg}$ de FGA (Acetato de Fluorogestona)', as quais foram depositadas na porção cranial da vagina, onde permaneceram por 11 dias. No nono dia do tratamento progestágeno os animais receberam, por via intramuscular, $50 \mu \mathrm{g}$ de Cloprostenol $^{2}$ e $400 \mathrm{UI}$ de eCG ${ }^{3}$ (Gonadotrofina Coriônica Eqüina). A partir de 12 horas da retirada das esponjas, foi

\footnotetext{
1 Synchro Part, Sanofi, França.

2 Ciosin, Coopers Brasil Ltda., Brasil.

${ }^{3}$ Novormon, Syntex S. A., Argentina.
}

realizada a detecção do estro a intervalos de quatro horas até que a última cabra apresentasse sinais de estro. Foram utilizados três reprodutores da raça Saanen para a realização da monta natural, a qual ocorreu em dois momentos: no início do estro e 24 horas após.

Os diagnósticos de gestação foram realizados, sempre pela mesma pessoa, aos 30, 45, 60, 75, 90, 105 e 120 dias após a monta natural. Foi utilizado um equipamento baseado no efeito Doppler, ${ }^{4}$ munido de um transdutor transretal de 2,2 $\mathrm{MHz}$. O reto da cabra, previamente esvaziado pela retirada das fezes, era lubrificado com gel ${ }^{5}$ a fim de melhorar o contato entre a mucosa retal e o transdutor, o qual era introduzido por aproximadamente $20 \mathrm{~cm}$ (Figura 1). Foi preestabelecido 0 tempo máximo de cinco minutos para realização de cada exame. Paralelamente, foram realizados exames com equipamento de ultra-sonografia em tempo real ${ }^{6}$ munido de um transdutor transabdominal de $3,5 \mathrm{MHz}$ (Figura 1).

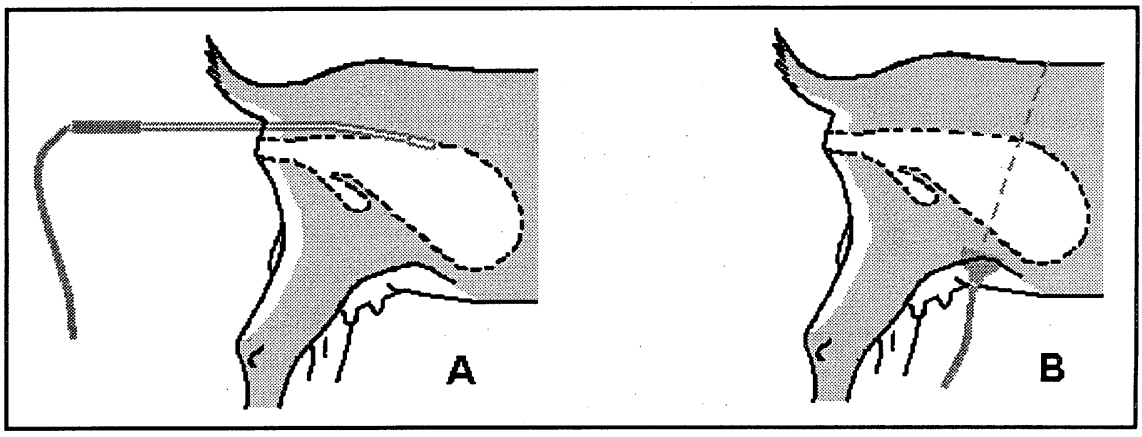

Figura 1 - Abordagem para o diagnóstico de gestação na cabra, utilizando-se os equipamentos baseados no efeito Doppler (A) e na ultra-sonografia em tempo real (B).

Para os dois métodos utilizados, efeito Doppler e ultrasonografia em tempo real, foi registrado o percentual de acerto para "gestação" e para "não-gestação", o percentual de acerto para gestação positiva baseado no número de crias nascidas, bem como, o número de batimentos cardíacos fetais e maternos quando do uso do efeito Doppler nas diferentes idades gestacionais. Utilizou-se o teste do Qui-quadrado para comparação dos percentuais de acerto de gestação e não-gestação entre os métodos, bem como para os percentuais de gestação positiva em relação ao número de crias nascidas. A comparação entre médias foi realizada através de análise de variância a um fator aplicando-se o teste PLSD de Fisher. Todos os testes estatísticos foram realizados utilizando o software Statview 5.0 (1998).

\section{Resultados e discussão}

Uma cabra foi retirada do experimento por não apresentar estro após o tratamento hormonal e, por conseguinte, não poder ser fecundada. Para as fêmeas restantes, o início do comportamento de estro ocorreu em média ( \pm d.p.) $25,8 \pm$ 10,8 horas após a retirada das esponjas. Esses resultados são semelhantes aos obtidos por Freitas et al. (1997) trabaIhando com animais da mesma raça e utilizando o mesmo tipo de tratamento hormonal.

\footnotetext{
4 DPPR-80, Microen, Brasil.

${ }^{5}$ Gel Carci, Pointer Química Industrial Ltda., Brasil.

${ }^{6}$ Shimadzu SDL-32, Japão.
} 
No que se refere ao percentual de acerto para gestação positiva, foram verificadas diferenças estatísticas entre os métodos aos 30, 45, 60, 75 e 105 dias de gestação, sendo que o método de ultra-sonografia em tempo real apresentou resultados superiores em todos as idades gestacionais. Comparando-se a eficiência do efeito Doppler, entre as diferentes idades gestacionais, verificou-se um baixo percentual de acerto aos 30 e 45 dias de gestação ( $0 \%$ e $22,2 \%$, respectivamente) e aumentando $(P<0,05)$ este percentual a partir dos 60 dias de gestação $(66,7 \%)$. Na mesma comparação, utilizando-se a ultrasonografia em tempo real, verificou-se não existirem diferenças estatísticas a partir dos 45 dias de gestação $(100,0 \%)$. Andrioli et al. (1997) verificaram, em cabras da raça Saanen, uma eficiência de $100 \%$ no diagnóstico de gestação por efeito Doppler aos 60 dias de idade gestacional, enquanto Goel e Agrawal (1990) encontraram $90 \%$ nas idades gestacionais de 51 a 60 dias. Esses resultados mostram-se superiores àqueles obtidos no presente estudo, porém deve-se salientar que a duração do exame é um fator que interfere em sua eficiência. Em nosso estudo, estabeleceu-se uma duração máxima de cinco minutos para cada exame, levando-se em consideração que um método de diagnóstico de gestação eficaz deve também apresentar-se como de fácil e rápida execução. Os dados encontrados no presente estudo, referentes à ultra-sonografia em tempo real, são similares aos citados anteriormente por outros autores nesta espécie (Bretzlaff et al., 1993; Doizé et al., 1997).

Nas diversas idades gestacionais não foi verificado nenhum resultado falso-positivo, isto é, um elevado percentual de acerto para não-gestação, quer seja para o efeito Doppler ou para ultra-sonografia em tempo real. O efeito Doppler apresentou resultados falsos-negativos aos $30(69,2 \%), 45(53,9 \%), 60(23,1 \%), 75$ $(23,1 \%), 90(15,4 \%), 105(23,1 \%)$ e 120 dias $(7,7 \%)$ de gestação. Esse fenômeno ocorreu, quando do uso da ultra-sonografia em tempo real, apenas aos 30 dias de gestação $(23,1 \%)$.

De acordo com Andrioli et al. (1997), o pequeno percentual de acerto para o diagnóstico de gestação por efeito Doppler, bem como o elevado percentual de resultados falsos negativos verificados aos 30 e 45 dias, podem estar relacionados ao pequeno diâmetro cardíaco dos fetos caprinos, dificultando a propagação das ondas sonoras das câmaras cardíacas e diminuindo o alcance do Doppler.
No que se refere ao número de fetos, verificou-se na ultrasonografia em tempo real um maior percentual de acerto de gestação $(P<0,05)$ apenas aos 30 dias de idade gestacional para cabras com gestação múltipla em relação àquelas de gestação simples $(44,4 \%$ vs $22,2 \%$, respectivamente). Para esse parâmetro, quando do uso do efeito Doppler, foram verificadas diferenças estatísticas $(P<0,05)$ aos $90(55,6 \%$ vs $22,2 \%)$ e aos $120(55,6 \%$ vs $33,3 \%)$ dias de idade gestacional em cabras com gestação múltipla ou simples, respectivamente. Não foram verificadas diferenças significativas entre gestação múltipla ou simples, em relação ao número de fetos, aos 105 dias de gestação. Este resultado deveu-se a somente um resultado falso negativo pelo efeito Doppler, podendo ser explicado pelo curto intervalo de tempo para a realização do exame, dificultando o diagnóstico de gestação (Figura 2).

Em nenhuma idade gestacional foi verificada diferença significativa nas freqüências cardíacas fetais, tanto para gestações simples quanto para gestações múltiplas (Tabela 1). Em cabras leiteiras, Olsson et al. (2001) mostraram que os batimentos cardíacos maternos foram elevados durante a

Tabela 1 - Média ( \pm d.p.) dos batimentos maternos (BM) ou fetais (BF) por minuto através do uso do efeito Doppler em várias idades gestacionais

\begin{tabular}{|c|c|c|c|c|c|c|c|c|c|c|c|c|c|c|}
\hline \multirow{3}{*}{$\begin{array}{l}N^{\circ} \\
\text { de } \\
\text { Fetos }\end{array}$} & \multicolumn{14}{|c|}{ Dias Após a Monta Natural } \\
\hline & \multicolumn{2}{|l|}{30} & \multicolumn{2}{|c|}{45} & \multicolumn{2}{|c|}{60} & \multicolumn{2}{|c|}{75} & \multicolumn{2}{|c|}{90} & \multicolumn{2}{|c|}{105} & \multicolumn{2}{|c|}{120} \\
\hline & BM & BF & BM & BF & BM & BF & BM & BF & BM & BF. & BM & BF & $B M$ & BF \\
\hline 1 & $89,0 \pm 10,0$ & $\cdot$ & $88,0 \pm 9,8$ & $142,0 \pm 0,0$ & $81,3 \pm 12,2$ & $206,7 \pm 11,4$ & $84,0 \pm 21,2$ & $200,0 \pm 18,3$ & $86,0 \pm 2,8$ & $220,0 \pm 25,5$ & $90,7^{a} \pm 4,6$ & $196,0 \pm 13,9$ & $81,3^{a} \pm 2,3$ & $200,0 \pm 34,6$ \\
\hline$>1$ & $97,4 \pm 19,9$ & $\cdot$ & $92,8 \pm 13,7$ & $228,0 \pm 0,0$ & $90,7 \pm 12,9$ & $190,0 \pm 17,3$ & $97,3 \pm 12,2$ & $236,0 \pm 60,4$ & $104,8 \pm 12,1$ & $203,2 \pm 14,8$ & $114,7^{\infty} \pm 12,2$ & $202,0 \pm 9,2$ & $104,4^{b} \pm 9,2$ & $210,4 \pm 22,2$ \\
\hline Total & $96,2 \pm 18,2$ & - & $90,2 \pm 11,9$ & $185,0 \pm 60,8$ & $85,2 \pm 12,2$ & $198,0 \pm 15,9$ & $92,9 \pm 16,0$ & $218,0 \pm 44,5$ & $97,2 \pm 13,4$ & $208,6 \pm 18,4$ & $100,6 \pm 15,4$ & $199,0 \pm 11,0$ & $91,8 \pm 13,3$ & $206,5 \pm 25,6$ \\
\hline
\end{tabular}

Médias seguidas por letras distintas, na mesma coluna, diferem estatisticamente $(P<0,05)$. 
Observou-se uma maior freqüência cardíaca materna nas cabras com gestação múltipla aos 105 e 120 dias de idade gestacional, quando comparadas com as de gestação simples. Este fato pode ser devido a uma maior necessidade de aporte sanguíneo na fase final de gestação, particularmente no caso de gestações múltiplas.

\section{Réferências}

ANDRIOLI, A.; BISCEGLI, C.I.; SOARES, A. T.; MOURA SOBRINHO, P. A. Detector de prenhez por efeito Doppler para caprinos. Rev. Bras. Reprod. Anim., v. 21, p. 148-149, 1997.

BARKER, C. A. V.; CAWLEY, A. J. Radiographic detection of fetal numbers in goats. Can. Vet. J., v. 8, p. 59-61, 1967.

BRETZLAFF, K.; EDWARDS, J.; FORREST, D., NUTI, L. Ultrasonographic determination of pregnancy in small ruminants. Vet. Med., v. 88, p. 1224, 1993.

CALLAHAN, D. A.; ROWLAND, T. C.; GOLDMAN, D. E. Ultrasonic Doppler observation of the fetal heart. Obst. Gynaecol., v. 23, p. 637640, 1964.

DOIZÉ, F.; VAILLANCOURT, D.; CARABIN, H.; BÉLANGER, D. Determination of gestational age in sheep and goat using transretal ultrasonographic measurement of placetomes. Theriogenology., v. 48, p. 449-460, 1997.

ENGELAND, I. V.; ROPSTAD, E.; ANDRESEN, EE.; EIK, L. O. Pregnancy diagnosis in dairy goats using progesterone assay kits and oestrus observation. Anim. Reprod. Sci.,v. 47, p. 237-243, 1997.

FREITAS, V.J. F.; BARIL, G.; SAUMANDE, J. Estrus synchronization in dairy goats: use of fluorogestone acetate vaginal sponges or norgestomet ear implants. Anim. Reprod. Sci., v. 46, p. 237-244, 1997.

GREYLING, J.P.C.; VANDERNEST; SCHWALBACH, L. M. J.; MULLER, $T$. Superovualtion and embryo transfer in South African Boer and Indigenous feral goats. Small Rum. Res., v. 43, p. 45-51, 2002.
Em conclusão, comparado à ultra-sonografia em tempo real, o diagnóstico de gestação em caprinos pelo uso do efeito Doppler torna-se interessante a partir dos 60 dias pós-fecundação. Além disso, a presença de gestação múltipla melhora a eficiência do método em final de gestação. Finalmente, o diagnóstico de gestação através do efeito Doppler é um método eficaz para utilização no manejo reprodutivo de caprinos, desde que seja conhecida a data de cobertura ou inseminação.

GOEL, A. K.; AGRAWAL, K. P. 1990. Pregnancy diagnosis in goats. Indian j. Vet. Med. v. 14, p.47-48.

ISHWAR, A. K. Pregnancy diagnosis in sheep and goats: a review. Small Rum. Res.,v. 17, p. 37-44, 1995.

OLSSON, K.; HANSSON, K.; HYDBRING, E.; von WALTER, L.W.; HAGGSTROM, J. A serial study of heart function during pregnancy, lactation and the dry period in dairy goats using echocardiography. Experim. Phys., v. 86, p. 93-99, 2001.

OTT, R. S.; BARUN, W. F.; LOCK, T. F.; MEMON, M. A.; STOWATER, J. L. A comparison of intraretal Doppler and rectal abdominal palpation for pregnancy testing in goats. J. Am. Vet. Med. Assoc., v. 178, p. 730731, 1981.

REFSTAL, K. R.; MARTENIUK, J. V.; WILLIAMS, C. S. F.; NACHREINER, R. F. Concentration of esterone sulphate in peripheral serum of pregnancy goats: relationship with gestation length, fetal number and the occurrence of fetal death in utero. Theriogenology., v. 36, p. 449-461, 1991.

RUSSEL, A. J. F. The application of real-time ultrasonic scanning to the diagnosis of pregnancy and determination of foetal number in goats. $J$. Goat Vet. Soc., v. 11, p. 9-14, 1990.

SMITH, M. C. Caprine reproduction. In: Morrow , D. A. (Ed.). Current Therapy in Theriogenology. 1. ed. 1980. p. 975-977.

SOUSA, N.M.;GARBAYO, J.M.; FIGUEIREDO,J. R.; SULON, J; GONÇALVES, P. B. D.; BECKERS, J. F. Pregnancy-associated glycoprotein and progesterone profiles during pregnancy and post partum in native goats from the northeast of Brazil. Small Rum. Res., v. 32, p. 137-147, 1999. STATVIEW 5.0. Statiview Reference. Cary: SAS Institute, 1998. 